\title{
Emerging Strategies to Enhance Homing and Engraftment of Hematopoietic Stem Cells
}

\author{
Mariusz Z. Ratajezak $^{1,2}$ - Malwina Suszynska ${ }^{1,2}$ \\ Published online: 24 September 2015 \\ (C) The Author(s) 2015. This article is published with open access at Springerlink.com
}

\begin{abstract}
Successful clinical outcomes from transplantation of hematopoietic stem cells (HSCs) depend upon efficient HSC homing to bone marrow (BM), subsequent engraftment, and, finally, BM repopulation. Homing of intravenously administered HSCs from peripheral blood (PB) through the circulation to the BM stem cell niches, which is the first critical step that precedes their engraftment, is enforced by chemotactic factors released in the $\mathrm{BM}$ microenvironment that chemoattract HSCs. These chemotactic factors include $\alpha$ chemokine stromal-derived factor 1 (SDF-1), the bioactive phosphosphingolipids sphingosine-1-phosphate (S1P) and ceramid-1-phosphate (C1P), and the extracellular nucleotides ATP and UTP. Stem cells may also respond to a $\mathrm{Ca}^{2+}$ or $\mathrm{H}^{+}$ gradient by employing calcium- or proton-sensing receptors, respectively. In this review, we will present emerging strategies based on ex vivo manipulation of graft HSCs that are aimed at enhancing the responsiveness of HSCs to BMsecreted chemoattractants and/or promoting HSC adhesion and seeding efficiency in the BM microenvironment.
\end{abstract}

Keywords Stem cell homing · Adult stem cells $\cdot$ CXCR4 . VLA-4 $\cdot$ SDF-1 $\cdot$ S1P $\cdot$ C1P $\cdot$ Extracellular nucleotides $\cdot$ Lipid rafts $\cdot$ Priming $\cdot$ Chemotaxis

Mariusz Z. Ratajczak

mzrata01@louisville.edu

1 Stem Cell Institute at the James Graham Brown Cancer Center, University of Louisville, 500 S. Floyd Street, Rm. 107, Louisville, KY 40202, USA

2 Department of Regenerative Medicine, Medical University of Warsaw, Warsaw, Poland

\section{Introduction}

After intravenous infusion, hematopoietic stem/progenitor cells (HSPCs) home through the circulation from peripheral blood (PB) to the bone marrow (BM) stem cell niches in response to chemoattractants secreted in the BM microenvironment, and this process precedes their subsequent engraftment and repopulation of the recipient's hematopoietic organs [1-3]. It is well known that hematopoietic recovery after transplantation of HSPCs and the final clinical outcome depend on the number and quality of HSPCs present in a graft, which can be estimated in humans by calculating the number of mononuclear cells that express the CD34 antigen. Based on this method, it has been determined that, for transplantation of umbilical cord blood (UCB) with $\leq 2$ human leucocyte antigen (HLA) disparities, the patient has to be infused with $\geq 2 \times 10^{5}$ UCB-derived $\mathrm{CD} 34^{+}$cells $/ \mathrm{kg}$ body weight [4]. When adult sources of HSPCs are employed (e.g., mobilized autologous $\mathrm{PB}), 2.5 \times 10^{6} \mathrm{CD} 34^{+}$cells $/ \mathrm{kg}$ body weight is considered a sufficient dose for successful stem cell autotransplant; however, a dose of $5.0 \times 10^{6} \mathrm{CD} 34^{+}$cells $/ \mathrm{kg}$ is considered preferable for achieving early engraftment [5].

These numbers point to the fact that hematopoietic reconstitution and recovery of normal PB counts after hematopoietic transplantation depends on the number of infused HSPCs. On the other hand, it is well known that not all HSPCs infused into the circulation find their way to the stem cell niches in $\mathrm{BM}$, and the majority is trapped in different nonhematopoietic locations in various organs. Therefore, it is important to develop more efficient strategies that improve the seeding efficiency of HSPCs by transplanting them directly to the $\mathrm{BM}$ microenvironment $[6,7]$. This is a very important issue, in particular when the number of HSPCs in the graft is low, as seen, for example, in adult recipients of UCB when there are low numbers of $\mathrm{CD} 34^{+}$cells harvested from $\mathrm{BM}$, or 
as a result of poor HSPC donor mobilization [6-8]. In all these cases, it is crucial to promote proper homing of HSPCs and thus ensure that as many $\mathrm{CD} 34^{+}$cells as possible home to the $\mathrm{BM}$ and subsequently permanently engraft.

One of the major mechanisms that retains HSPCs in their $\mathrm{BM}$ niches and directs their migration and homing from $\mathrm{PB}$ to $\mathrm{BM}$ involves interaction of the CXCR4 receptor with $\alpha$ chemokine stromal-derived factor 1 (SDF-1). While CXCR4 is expressed on the surface of HSCs, SDF-1 is expressed on the surface of cells lining the stem cell niches [1-3, 9]. Homing is also orchestrated by gradients of other chemotactic factors that show chemotactic activity against HSPCs. The list of these chemoattractants is rather short, and so far it has been demonstrated that, besides SDF-1, HSPCs respond to gradients of sphingosine-1-phosphate (S1P) [10-14], ceramide-1phosphate (C1P) [12], certain extracellular nucleotides, such as ATP or UTP [15], as well as certain ions, such as $\mathrm{Ca}^{2+}$ and $\mathrm{H}^{+}[16,17]$.

In this review we present emerging strategies aimed at improving the responsiveness of HSPCs to homing gradients as well as strategies to increase the tethering of transplanted HSPCs to the BM endothelium and subsequently their adhesion in the BM microenvironment. In order to focus on this particular topic, we will not discuss other strategies, such as ex vivo expansion of HSPCs to be used in a graft or application of allo-engraftment-facilitating cells. These strategies that also lead to better engraftment of transplanted HSPCs were recently reviewed elsewhere in excellent publications [18, 19].

We will review various strategies for improving the homing and engraftment of HSPCs (Fig. 1), based on their classification into the following categories: i) increasing the biological effects of membrane lipid rafts, ii) modifying the expression and function of BM homing molecules, iii) modifying the metabolism of HSPCs, and iv) enhancing the availability of chemotactic factors for HSPCs. However, we are aware that this is a rather artificial classification of the strategies employed in this review and is followed for reasons of simplicity; however, several of these strategies, in fact, overlap and involve more than one category.

\section{Homing Strategies Based on Increasing the Biological Effects of Membrane Lipid Rafts}

The cell surface membrane is a selectively permeable structure that separates the interior of the cell from the outside environment surrounding the cell. The cell membrane consists of a phospholipid bilayer, with several embedded proteins, that is held together via non-covalent interactions between the hydrophobic phospholipid tails. It has been demonstrated that, under physiological conditions, phospholipid molecules in the cell membrane are in a liquid crystalline state. However, cell membranes also contain combinations of glycosphingolipids and protein receptors organized into glycoprotein microdomains, called lipid rafts, which play an important role in several processes regulating cell biology [20,21]. These dynamic microscopic cholesterol-enriched structures are important in assembling signaling molecules together with cell surface receptors and have been identified as playing a primary role in signaling. For example, the SDF-1-binding, G proteincoupled receptor CXCR4 must be incorporated into membrane lipid rafts for optimal association with downstream signaling proteins [22]. Thus, strategies aimed at promoting lipid raft formation could enhance the responsiveness of HSPCs to SDF-1 gradients and thereby facilitate homing of HSPCs (Fig. 1a).

The Priming Effect of Certain Small Molecules That Increase the Responsiveness of HSCs to an SDF-1 Gradient The SDF-1-CXCR4 axis is the most important chemotactic axis involved in the homing of HSPCs to BM [1-3]. Nevertheless, the measured SDF-1 level in the BM microenvironment after myeloblative conditioning for transplantation is relatively low $(2-3 \mathrm{ng} / \mathrm{ml})$. This level is in striking contrast to the SDF-1 doses usually employed $(200-300 \mathrm{ng} / \mathrm{ml})$ in ex vivo experimental migration assays for HSPCs, which are much too high and not physiologic. Moreover, although SDF1 mRNA increases in the BM microenvironment after myeloablative conditioning by radio- or chemotherapy [23], this treatment induces a highly proteolytic microenvironment in BM [24]. SDF-1, as a peptide susceptible to proteolytic digestion, is a target for several proteolytic enzymes that are released from myeloid cells, and thus it becomes easily digested and inactivated [25].

Nevertheless, evidence has accumulated that this potential decrease in SDF-1 protein level in the BM microenvironment after conditioning for transplantation is somewhat mitigated by several factors, such as complement cascade (ComC) cleavage fragments ( $\mathrm{C} 3 \mathrm{a}$ and ${ }_{\text {desArg }} \mathrm{C} 3 \mathrm{a}$ ), other cationic microbial peptides released from granulocytes, such as cathelicidin (LL-37) and $\beta 2$-defensin, as well as other molecules, such as hyaluronic acid, fibronectin, and soluble VCAM-1, ICAM-1, and UPAR, which may significantly enhance the responsiveness of HSPCs to a shallow SDF-1 gradient [3, 21, 22, 26-28]. This phenomenon is known in the literature as a priming effect on an SDF-1 gradient and significantly compensates for a decrease in SDF-1 level in the BM microenvironment, which occurs due to its degradation after induction of a proteolytic microenvironment. The priming effect is explained by evidence that all these small molecules promote lipid raft formation in HSPCs and thus increases HSPC responsiveness to an SDF-1 gradient via recruitment of CXCR4 [29]. Therefore, short ex vivo exposure of HSPCs to certain small molecules before infusion enhances their homing to BM. This effect has been demonstrated in vivo with C3a [30] and LL-37 [26]. Using several experimental strategies, including confocal 
Fig. 1 Currently proposed strategies that may improve homing and engraftment of HSPCs. a Strategies based on increasing the biological effects of membrane lipid rafts. b Modification of the expression and function of molecules involved in adhesion and BM homing. c Metabolic modification of the homing properties of HSPCs. d Enhancing the bioavailability of chemotactic factors for HSPCs. This classification is presented for reasons of simplicity; however, it is clear that several of the proposed strategies have overlapping effects affecting other mechanisms

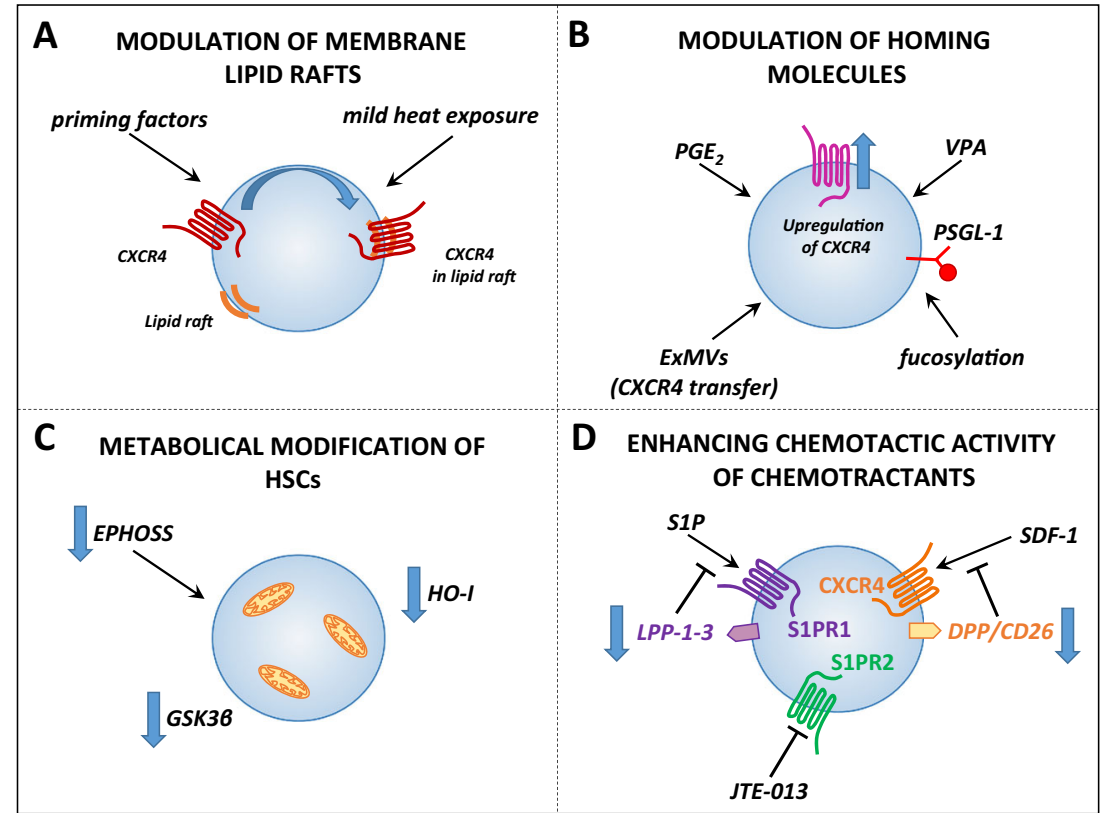

microscopy and analysis of membrane fractions enriched in lipid rafts, it has been demonstrated that, after exposure of HSPCs to these small molecules, CXCR4 (a membrane lipid raft-associated receptor) interacts more efficiently with SDF-1 and better engages proteins in downstream signaling pathways that are involved in trafficking and adhesion of HSPCs $[22,31,32]$.

The feasibility of ex vivo C3a priming of UCB cells before infusion into the patient was recently demonstrated in a clinical trial [33]. In the meantime, however, experimental evidence has accumulated that LL-37, a cathelicidin fragment, is a much more potent priming factor than C3a [26]. Moreover, since LL-37 is a physiological antibiotic peptide secreted by granulocytes and a potentially safe molecule for ex vivo graft manipulation, we propose an LL-37-based priming strategy as an interesting and simple alternative.

Short Exposure of HSPCs to Mild Heat Treatment Another interesting strategy to increase lipid raft formation on the surface of HSPCs, and thus facilitate homing and engraftment of these cells, has been proposed recently by another group [32]. Specifically, it was demonstrated that a short, mild ex vivo heat treatment $\left(39.5^{\circ} \mathrm{C}\right)$ of a UCB graft primed the human $\mathrm{CD} 34^{+}$cells for migration in response to an SDF-1 gradient and enhanced engraftment of these cells in immunodeficient mice.

This mild heat treatment was associated with increased expression of CXCR4 on human $\mathrm{CD} 34^{+}$cells and, most importantly, enhanced co-localization of CXCR4 within the lipid raft domains. CXCR4 incorporated in lipid rafts interacted better with Rac-1, a GTPase that is crucial for cell migration and adhesion [32]. Based on this intriguing observation, mild heating of umbilical cord blood-derived HSPCs before transplantation may, as proposed by the authors, become a relatively simple and inexpensive strategy to enhance homing and engraftment of transplanted cells.

\section{Modification of Expression and Function of BM Homing Molecules}

There are several molecules on the surface of HSPCs that play a role in directing cell migration in response to BM-released chemoattractants, tethering of infused cells to the endothelium in BM sinusoids, and promoting their subsequent adhesion in the BM microenvironment. The most important receptors, besides the already mentioned CXCR4, are the type 1 receptor for S1P $\left(\mathrm{S}_{1} \mathrm{PR}_{1}\right)$ and certain cell-surface mucins (e.g., Pselectin glycoprotein ligand 1 [PSGL-1]) [34]. Based on these findings, some of the strategies to improve homing have been developed based, on the one hand, on increased expression of these receptors on the cell surface and, on the other hand, on enhancing their biological pro-adhesive functions (Fig. 1b).

Pulse Exposure of HSPCs to Prostaglandin $\mathbf{E}_{\mathbf{2}}$ Prostaglandins are very short-lived molecules that belong to a large family of bioactive lipids. All nucleated cells, including those in the BM microenvironment, synthesize prostaglandins, which are involved in several pleiotropic biological effects. Prostaglandin $\mathrm{E}_{2}\left(\mathrm{PGE}_{2}\right)$ has been reported to be involved in regulating the biology and trafficking of HSPCs [35]. It has been shown that pulse exposure of murine or human BM cells to $\mathrm{PGE}_{2}$ stimulates cell proliferation, cycling, and differentiation of more primitive hematopoietic stem cells (HSCs), leading to 
an increase in the number of more differentiated hematopoietic progenitor cells (HPCs) [35]. These observations obtained with murine and human cells were recently validated in a zebrafish model [36]. Based on these observations, a shortpulse exposure to $\mathrm{PGE}_{2}$ was employed to facilitate engraftment of murine and human HSPCs. As reported, one of the effects of PGE2 is to increase expression of CXCR4 on the surface of HSPCs, which facilitates their responsiveness to SDF-1 gradients. Pre-clinical limiting-dilution studies revealed that equivalent engraftment in immunodeficient mice was achieved with 4-fold fewer $\mathrm{PGE}_{2}$-treated human UCB cells than with untreated control UCB cells [37]. In sum, the effect of $\mathrm{PGE}_{2}$ in upregulating CXCR4 receptor expression plays an important role, on the one hand, in their migratory responsiveness to an SDF-1 gradient and, on the other hand, in tethering infused HSPCs to SDF-1 expressed on the surface of endothelial cells in BM sinusoids [38]. This promising strategy has already been confirmed in a clinical study and awaits further validation [6].

\section{Upregulation of the CXCR4 Receptor on the Surface of} HSPCs by Valporic Acid It is obvious that any strategy that leads to upregulation of CXCR4 on the surface of HSPCs would enhance the homing effect of the SDF-1-CXCR4 axis. While potential application of CXCR4-overexpressing vectors in HSPCs is problematic because of safety issues, a relatively simple strategy has been proposed based on exposure of HSPCs to the histone deacetylase inhibitor valporic acid (VPA) [39]. It is well known that histone deacetylase inhibitors, including VPA, stimulate proliferation and self-renewal of normal HSPCs. Importantly, it has been demonstrated that exposure of murine BM mononuclear cells to VPA enhanced spleen colony formation and BM engraftment of murine HSPCs. To shed more light on this phenomenon, human UCB-derived $\mathrm{CD} 34^{+}$cells were exposed to VPA, and it has been observed that such exposure both upregulates CXCR4 expression on the surface of HSPCs and enhances migration of these cells up an SDF-1 gradient [39].

This relatively simple strategy to expose ex vivo HSPs to VPA has become important, because VPA has recently been successfully employed for expansion of UCB-derived primitive HSCs, which are enriched for Oct- $4^{+}$cells [40]. Therefore, the combined effects of VPA on upregulation of CXCR4 on the cell surface to enhance cell homing and on ex vivo expansion of HSCs could become a promising strategy for clinical application of this drug.

Surface Fucosylation of HSPCs As mentioned above, after infusion into the circulation, HSPCs navigate towards the BM, and the first step in the homing process is their rolling adhesions and tethering on P-selectin and E-selectin, which are expressed on the surface of endothelial cells in BM sinusoids [34]. The P- and E-selectins expressed by endothelial cells are membrane-bound lectins that interact with cell-surface glycoconjugate ligands expressed on the surface of HSPCs. In order to be biologically active, these selectin ligands have to be properly $\alpha 1-3$ fucosylated [34].

The most important ligand for P- and E-selectins on endothelial cells in BM sinusoids is the HSPC-expressed mucin known as P-selectin glycoprotein ligand 1 (PSGL-1). It has been reported in the case of UCB-derived HSPCs that $\alpha(1,3)$ fucosylation of mucins on the surface of these cells is inadequate and results in poor binding of $\mathrm{CD} 34^{+} \mathrm{CD} 38^{- \text {llow }}$ cells to E- and P-selectins in BM sinusoids [34]. To ameliorate this defect, UCB cells were exposed to guanosine diphosphate fucose in the presence of $\alpha(1,3)$ fucosyltransferase VI in order to fucosylate cell surface mucins, including PSGL-1. It has been reported that this ex vivo enzymatic approach enhanced engraftment of UCB cells in BM in immunodeficient NOD/ SCID mice [34]. Nevertheless, this interesting strategy awaits verification in a clinical setting.

"Painting" of HSPCs by Platelet-Derived Extracellular Microvesicles Another approach that has been proposed to improve tethering of transplanted HSPCs to the BM endothelium is ex vivo incubation of the HSPCs in a hematopoietic graft with PB platelet-derived extracellular microvesicles (ExMVs) [41]. It has been demonstrated that ExMVs transfer not only CXCR4 but also several receptors that are crucial for adhesion of platelets to endothelium from platelets to HSPCs. Based on this effect, HSPCs isolated from murine BM or human UCB and pre-incubated with platelet-derived ExMVs engrafted much faster after transplantation into normal or immundeficient mice, respectively [41]. Furthermore, since HSPCs isolated from mobilized PB are already highly covered by platelet-derived ExMVs as a result of platelet activation in the plastic tubing during leucopheresis, this strategy is more suitable for HSPCs aspirated from BM or isolated from UCB. It has been hypothesized that, since HSPCs isolated from mobilized PB are already densely covered by ExMVs, this phenomenon may explain differences in engraftment kinetics between mPB-derived and BM-aspirated HSPCs.

\section{Metabolic Modification of HSPCs}

The knowledge and identification of metabolic pathways that regulate self-renewal, proliferation, differentiation, and migration of HSPCs has increased significantly over the past few years. In parallel, the role of mitochondria in HSPC biology as a source of free radicals [42], the biological effects of heme oxygenase 1 [43], and the involvement of glycogen synthase kinase $3 \beta$ (GSK- $3 \beta)$ as a regulator of the wingless (Wnt) $-\beta$ catenin pathway are better understood $[44,45]$. Based on this enhanced understanding, several new strategies to improve homing and engraftment have been proposed (Fig. 1c). 
Exposure of Cells in Hematopoietic Grafts to Hypoxia as a Means to Enhance Their Homing and Engraftment It is known that HSPCs reside in specific BM stem cell niches under hypoxic conditions and rely for their metabolism on anaerobic glycolysis. As in BM, HSPCs in UCB are exposed to hypoxia. By contrast, most of the current processing strategies to prepare hematopoietic grafts from BM- or UCBderived cells are performed under hyperoxic conditions, which leads to induction of transition pore permeability in mitochondria and results in the release of free radicals or reactive oxygen species (ROS) [42].

ROS, on the other hand, increase differentiation and cycling of HSCs, which may result in their differentiation and depletion. This effect of hyperoxia combined with release of ROS from mitochondria has been named extraphysiologic oxygen shock/stress (EPHOSS) [42]. This unwanted effect of ROS release can be prevented by collection and processing of HSPCs in low oxygen tension (3\%) in ambient air or in the presence of cyclosporin A (CyA), which protects cells from EPHOSS. In support of this concept, a beneficial effect of hypoxia and CyA was demonstrated in a recent elegant study employing an HSPC transplant model in mice [42]. This promising strategy may lead to initiation of a relatively simple, but at the same time very promising, clinical trial to enhance engraftment of UCB.

Inhibition of Heme Oxygenase 1 (HO-1) in HSPCs Heme oxygenase 1 (HO-1) is an inducible stress-response enzyme that not only catalyzes the degradation of heme (e.g., released from damaged erythrocytes) but also performs an important function in various physiological and pathophysiological states associated with cellular stress, such as ischemic/ reperfusion injury. As already reported, HO-1 has negative effects on adhesion and migration of neutrophils in the state of acute inflammation [46], and this observation was confirmed recently for HSPCs [43]. HSPCs purified from HO-1 $\mathrm{KO}$ mice, and thus $\mathrm{HO}-1$-deficient, show enhanced migration in response to SDF-1 and S1P gradients. This phenomenon is also supported by the highly migratory state in vivo of HSPCs from mice lacking one $\mathrm{HO}-1$ allele $\left(\mathrm{HO}-1^{+-}\right)$. Interestingly, wild type mice transplanted with $\mathrm{BM}$ from $\mathrm{HO}-1^{+/-}$animals showed accelerated hematopoietic recovery from myelotoxic injury compared with lethally irradiated recipients transplanted with BM cells from normal control animals [43]. Unfortunately, mice transplanted with HO- $1^{+/-}$HSPCs were less effective in radioprotection as well as in serial repopulation studies of myeloablated recipients.

Based on these observations, we hypothesized that transient but not permanent inhibition of HO-1, achieved by employing ex vivo exposure to small-molecule inhibitors of this enzyme, could have a beneficial effect in increasing the chemotactic responsiveness of HSPCs to SDF-1 and S1P homing gradients and that this $\mathrm{HO}-1$ transient inhibition strategy could enhance homing and engraftment of transplanted HSPCs [43]. In fact, our in vitro and in vivo animal experiments demonstrated that transiently inhibiting HO-1 activity in HSPCs by small-molecule inhibitors improves HSPC homing and engraftment. We propose that this simple and inexpensive strategy could be employed in the clinical setting to improve engraftment of HSPCs, particularly in those cases in which the number of HSPCs available for transplant is limited and optimal engraftment of infused cells is desirable.

Inhibition of GSK-3 $\beta$ in HSPCs to Improve Engraftment The main purpose of hematopoietic transplantation is to transplant long-term repopulating HSCs endowed with the ability to sustain long-term hematopoiesis. Glycogen synthase kinase $3 \beta$ (GSK-3 $\beta$ ) has been identified as an important regulator of HSC function due to activation of the wingless (Wnt)- $\beta$-catenin pathway, which stimulates proliferation of HSCs [44]. Thus, Wnt $-\beta$ catenin signaling plays an important role in maintaining stem cell regenerative potential under steadystate conditions. Wnt signaling enforces quiescence of HSCs in BM niches and preserves the capacity of these cells for selfrenewal. This mechanism is often perturbed in cells isolated from BM or UCB and, in particular, in HSPCs after their ex vivo expansion. Therefore, a novel strategy based on ex vivo application of the GSK-3 $\beta$ inhibitor, 6 bromoindirubin $3^{\prime}$ oxime (BIO), has been proposed to delay cycling of harvested cells so that long-term repopulating HSCs will be preserved in the graft [45]. In appropriate experimental models it has been convincingly demonstrated that GSK-3 $\beta$ inhibition promotes engraftment of ex vivoexpanded human and murine HSPCs in normal and immunodeficient mouse models, respectively [44, 45]. However, this strategy of employing a small-molecule inhibitor of GSK-3 $\beta$ has not yet been tested in a clinical setting.

\section{Enhancing the Availability of Chemotactic Factors for HSPCs}

HSPC chemotactic factors play a crucial role in BM homing and, as mentioned above, a proteolytic microenvironment induced by myeloablative conditioning affects the bioavailability of SDF-1 in BM. While we have learned a lot about induction of a proteolytic microenvironment in $\mathrm{BM}$ as a response to myeloablative conditioning, more work is needed to see whether, in addition to proteolytic enzymes, lipolytic enzymes are also induced and thereby affect the bioavailability of S1P and C1P. Similarly, enzymes degrading ATP could also potentially affect an ATP homing gradient. On the other hand, as will be discussed below, some of the enzymes that may affect the bioavailability of chemotactic factors are secreted by HSPCs themselves (Fig. 1d). 
Inhibition of Dipeptidylpeptidase 4 (DPP4) on the Surface of HSPCs Dipeptidylpeptidase 4 (DPP4, also known as CD26) antigen, expressed on the surface of HSPCs, is endowed with proteolytic activity and may inactivate SDF-1 close to the cell membrane before it engages the CXCR4 receptor [47]. To ameliorate this unwanted effect, it has been proposed that blockade of DPP4 enhances the responsiveness of HSPCs to an SDF-1 gradient. In support of this hypothesis, both competitive and non-competitive hematopoietic transplants of murine and human HSPCs in normal and immunodeficient mice, respectively, revealed that a short exposure of transplanted cells to diprotinin $\mathrm{A}$, which is an inhibitor of proteolytic DPP4 activity on the cell surface, enhanced homing and engraftment of these cells [47]. Thus, inhibition of DPP4 prevents SDF-1 degradation and facilitates its chemotactic interaction with the CXCR4 receptor. Most importantly, an oral inhibitor of DPP4 known as sitagliptin has been developed, and this form of DPP4 inhibitor is currently employed in clinical trials in humans transplanted with UCB [6]. This promising strategy awaits clinical verification with a larger number of patients.

\section{Modification of the Responsiveness of HSPCs to S1P and} C1P Gradients As mentioned above, bioactive phosphosphingolipids are potent chemoattractants for HSPCs in physiological doses and the cell-surface binding receptors for S1P are well described. These receptors are members of a

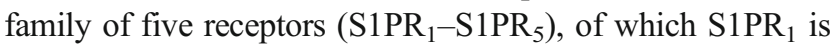
crucial in eliciting the chemotactic responsiveness of cells to an S1P gradient [29]. By contrast, $\mathrm{S}_{\mathrm{PR}}$ has the opposite function [29]. Therefore, one tempting strategy is to blockade $\mathrm{S}_{1} \mathrm{PR}_{2}$ by employing a specific small-molecule inhibitor, JTE013. This strategy has already been demonstrated to improve migration of neural progenitors in response to an S1P gradient [48].

HSPCs also express lipid phosphate phosphatases (LPP13 ) on the cell surface, which possess ecto-enzymaticdegrading activity against S1P and C1P [29, 49]. Thus, LPP1-3 expressed on the surface of HSPCs could negatively affect their migration in response to gradients of $\mathrm{C} 1 \mathrm{P}$ and $\mathrm{S} 1 \mathrm{P}$ (dephosphorylated sphingosine and ceramide do not chemoattract HSPCs). This is a somewhat analogous phenomenon to the effect of DPP4 on SDF-1-mediated chemotaxis. Therefore, in order to increase the responsiveness of HSPCs to $\mathrm{S} 1 \mathrm{P}$ and $\mathrm{C} 1 \mathrm{P}$ gradients, it would be interesting to see whether ex vivo exposure of HSPCs to the LLP inhibitor XY-14 enhances engraftment of HSPCs.

Finally, since the BM level of S1P may be additionally increased by inhibiting S1P lyase (SPL) by deoxypyridoxine (DOP), a vitamin B6 antagonist, or 2-acethyl-4tertrahydroxybutylimaidazole (THI), this inhibition should enhance the S1P level in BM and thus additionally improve the responsiveness of HSPCs to an S1P homing gradient [29].
Since inhibitors of S1P lyase (DOP and THI) are FDAapproved compounds, this relatively simple strategy for improving homing of HSPCs in patients that can be preconditioned before transplantation with an SPL inhibitor awaits experimental verification.

\section{Conclusions}

Optimal and efficient stem cell homing will also enable achievement of the desired therapeutic effect using a lower number of stem cells. This is important, for example, in transplantation of UCB. Since the number of HSCs in UCB is limited and often insufficient to transplant to an adult recipient, strategies that enhance homing (seeding efficiency of HSCs to BM) and subsequent engraftment of UCB-derived HSCs are vital for positive clinical outcomes. In the near future we expect that some of the strategies proposed above will be widely employed or that even more novel and powerful strategies will be developed to enhance stem cell homing that target both the local niche and the stem cells employed for treatment. In addition, it is also possible to combine some of the strategies for improved homing, as discussed above. In support of this notion, inhibition of DPP4 was successfully combined with a short exposure of human or murine HSPCs to $\mathrm{PGE}_{2}$ to enhance their engraftment in experimental animal models [38]. Moreover new factors become identified such as for example DEK protein [50] that may ex vivo stimulate hematopoietic graft and thus enhance homing and engraftment of HSPCs. Finally, efficient homing and engraftment of stem cells employed in therapy has an immediate impact on the final outcome of the therapy not only in hematological settings but also in other potential applications of stem cells in tissue or organ injuries (e.g., to myocardium, liver, or kidney) [51].

Acknowledgments This work was supported by NIH grants 2R01 DK074720 and R01HL112788 and the Stella and Henry Endowment to MZR.

\section{Compliance of Ethical Standards}

Conflict of interest The authors declare indicate no potential conflicts of interest.

Open Access This article is distributed under the terms of the Creative Commons Attribution 4.0 International License (http:// creativecommons.org/licenses/by/4.0/), which permits unrestricted use, distribution, and reproduction in any medium, provided you give appropriate credit to the original author(s) and the source, provide a link to the Creative Commons license, and indicate if changes were made. 


\section{References}

1. Lévesque, J. P., Helwani, F. M., \& Winkler, I. G. (2010). The endosteal 'osteoblastic' niche and its role in hematopoietic stem cell homing and mobilization. Leukemia, 24, 1979-1992.

2. Lapidot, T., \& Kollet, O. (2010). The brain-bone-blood triad: traffic lights for stem-cell homing and mobilization. Hematology American Society of Hematology. Education Program, 2010, 1-6.

3. Ratajczak, M. Z. (2015). A novel view of the adult bone marrow stem cell hierarchy and stem cell trafficking. Leukemia, 29, 776782.

4. Gluckman, E., \& Rocha, V. (2009). Cord blood transplantation: state of the art. Haematologica, 94, 451-454.

5. Reddy, R. L. (2005). Mobilization and collection of peripheral blood progenitor cells for transplantation. Transfusion and Apheresis Science, 32, 63-72.

6. Pineault, N., \& Abu-Khader, A. (2015). Advances in umbilical cord blood stem cell expansion and clinical translation. Experimental Hematology, 43, 498-513.

7. Broxmeyer, H. E. (2012). Enhancing engraftment of cord blood cells via insight into the biology of stem/progenitor cell function. Annals of the New York Academy of Sciences, 1266, 151-160.

8. Lund, T. C., Boitano, A. E., Delaney, C. S., Shpall, E. J., \& Wagner, J. E. (2015). Advances in umbilical cord blood manipulation-from niche to bedside. Nature Reviews. Clinical Oncology, 12, 163-174.

9. Ara, T., Tokoyoda, K., Sugiyama, T., Egawa, T., Kawabata, K., \& Nagasawa, T. (2003). Long-term hematopoietic stem cells require stromal cell-derived factor-1 for colonizing bone marrow during ontogeny. Immunity, 19, 257-267.

10. Seitz, G., Boehmler, A. M., Kanz, L., \& Möhle, R. (2005). The role of sphingosine 1-phosphate receptors in the trafficking of hematopoietic progenitor cells. Annals of the New York Academy of Science, 1044, 84-89.

11. Massberg, S., \& von Andrian, U. H. (2009). Novel trafficking routes for hematopoietic stem and progenitor cells. Annals of the New York Academy of Sciences, 1176, 87-93.

12. Ratajczak, M. Z., Lee, H., Wysoczynski, M., Wan, W., Marlicz, W., Laughlin, M. J., et al. (2010). Novel insight into stem cell mobilization-Plasma sphingosine-1-phosphate is a major chemoattractant that directs the egress of hematopoietic stem progenitor cells from the bone marrow and its level in peripheral blood increases during mobilization due to activation of complement cascade/membrane attack complex. Leukemia, 24, 976-985.

13. Juarez, J. G., Harun, N., Thien, M., Welschinger, R., Baraz, R., Pena, A. D., et al. (2012). Sphingosine-1-phosphate facilitates trafficking of hematopoietic stem cells and their mobilization by CXCR4 antagonists in mice. Blood, 119, 707-716.

14. Golan, K., Vagima, Y., Ludin, A., Itkin, T., Cohen-Gur, S., Kalinkovich, A., et al. (2012). S1P promotes murine progenitor cell egress and mobilization via S1P1-mediated ROS signaling and SDF-1 release. Blood, 119, 2478-2488.

15. Rossi, L., Manfredini, R., Bertolini, F., Ferrari, D., Fogli, M., Zini, R., et al. (2007). The extracellular nucleotide UTP is a potent inducer of hematopoietic stem cell migration. Blood, 109, 533-542.

16. Adams, G. B., Chabner, K. T., Alley, I. R., Olson, D. P., Szczepiorkowski, Z. M., Poznansky, M. C., et al. (2006). Stem Cell engraftment at the endosteal niche is specified by the calcium-sensing receptor. Nature, 439, 599-603.

17. Okajima, F. (2013). Regulation of inflammation by extracellular acidification and proton-sensing GPCRs. Cellular Signalling, 25, 2263-2271.

18. Colson, Y. L., Shinde Patil, V. R., \& Ildstad, S. T. (2007). Facilitating cells: novel promoters of stem cell alloengraftment and donor-specific transplantation tolerance in the absence of GVHD. Critical Reviews in Oncology/Hematology, 6, 26-43.
19. Walasek, M. A., van Os, R., \& de Haan, G. (2012). Hematopoietic stem cell expansion: challenges and opportunities. Annals of the New York Academy of Sciences, 1266, 138-150.

20. Goñi, F. M. (2014). The basic structure and dynamics of cell membranes: an update of the Singer-Nicolson model. Biochimica et Biophysica Acta, 1838, 1467-1476.

21. Ratajczak, M. Z., \& Adamiak, M. (2015). Membrane lipid rafts, master regulators of hematopoietic stem cell retention in bone marrow and their trafficking. Leukemia, 29, 1452-1457.

22. Wysoczynski, M., Reca, R., Ratajczak, J., Kucia, M., Shirvaikar, N., Honczarenko, M., et al. (2005). Incorporation of CXCR4 into membrane lipid rafts primes homing-related responses of hematopoietic stem/progenitor cells to an SDF-1 gradient. Blood, 105, 40 48.

23. Ponomaryov, T., Peled, A., Petit, I., Taichman, R. S., Habler, L., Sandbank, J., et al. (2000). Induction of the chemokine stromalderived factor-1 following DNA damage improves human stem cell function. The Journal of Clinical Investigation, 106, 1331-1339.

24. Lévesque, J. P., Hendy, J., Takamatsu, Y., Williams, B., Winkler, I. G., \& Simmons, P. J. (2002). Mobilization by either cyclophosphamide or granulocyte colony-stimulating factor transforms the bone marrow into a highly proteolytic environment. Experimental Hematology, 30, 440-449.

25. Kim, C. H., Wu, W., Wysoczynski, M., Abdel-Latif, A., Sunkara, M., Morris, A., et al. (2012). Conditioning for hematopoietic transplantation activates the complement cascade and induces a proteolytic environment in bone marrow: a novel role for bioactive lipids and soluble C5b-C9 as homing factors. Leukemia, 26, 106-116.

26. Wu, W., Kim, C. H., Liu, R., Kucia, M., Marlicz, W., Greco, N., et al. (2012). The bone marrow-expressed antimicrobial cationic peptide LL-37 enhances the responsiveness of hematopoietic stem progenitor cells to an SDF-1 gradient and accelerates their engraftment after transplantation. Leukemia, 26, 736-745.

27. Avigdor, A., Goichberg, P., Shivtiel, S., Dar, A., Peled, A., Samira, S., et al. (2004). CD44 and hyaluronic acid cooperate with SDF-1 in the trafficking of human CD34+ stem/progenitor cells to bone marrow. Blood, 103, 2981-2989.

28. Wysoczynski, M., Reca, R., Lee, H., Wu, W., Ratajczak, J., \& Ratajczak, M. Z. (2009). Defective engraftment of C3aR-/- hematopoietic stem progenitor cells reveals a novel role of the $\mathrm{C} 3 \mathrm{a}-\mathrm{C} 3 \mathrm{aR}$ axis in bone marrow homing. Leukemia, 23, 1455-1461.

29. Ratajczak, M. Z., Kim, C. H., Abdel-Latif, A., Schneider, G., Kucia, M., Morris, A. J., et al. (2012). A novel perspective on stem cell homing and mobilization: review on bioactive lipids as potent chemoattractants and cationic peptides as underappreciated modulators of responsiveness to SDF-1 gradients. Leukemia, 26, 63-72.

30. Ratajczak, M. Z., Reca, R., Wysoczynski, M., Kucia, M., Baran, J. T., \& Allendorf, D. J. (2004). Transplantation studies in C3deficient animals reveal a novel role of the third complement component $(\mathrm{C} 3)$ in engraftment of bone marrow cells. Leukemia, 18, 1482-1490.

31. Chae, H. D., Lee, K. E., Williams, D. A., \& Gu, Y. (2008). Crosstalk between RhoH and Rac1 in regulation of actin cytoskeleton and chemotaxis of hematopoietic progenitor cells. Blood, 111, 2597-2605.

32. Capitano, M. L., Hangoc, G., Cooper, S., \& Broxmeyer, H. E. (2015). Mild heat treatment primes human CD34+ cord blood cells for migration toward SDF- $1 \alpha$ and enhances engraftment in an NSG mouse model. Stem Cells, 33, 1975-1984.

33. Brunstein, C. G., McKenna, D. H., DeFor, T. E., Sumstad, D., Paul, P., Weisdorf, D. J., et al. (2013). Complement fragment 3a priming of umbilical cord blood progenitors: safety profile. Biology of Blood and Marrow Transplantation, 19, 1474-1479.

34. Xia, L., McDaniel, J. M., Yago, T., Doeden, A., \& McEver, R. P. (2004). Surface fucosylation of human cord blood cells augments 
binding to P-selectin and E-selectin and enhances engraftment in bone marrow. Blood, 104, 3091-3096.

35. Hoggatt, J., Singh, P., Sampath, J., \& Pelus, L. M. (2009). Prostaglandin E2 enhances hematopoietic stem cell homing, survival, and proliferation. Blood, 113, 5444-5455.

36. North, T. E., Babu, I. R., Vedder, L. M., Lord, A. M., Wishnok, J. S., Tannenbaum, S. R., et al. (2010). PGE2-regulated wnt signaling and $\mathrm{N}$-acetylcysteine are synergistically hepatoprotective in zebrafish acetaminophen injury. Proceedings of the National Academy of Sciences of the United States of America, 105, 17315-17320.

37. Pelus, L. M., Hoggatt, J., \& Singh, P. (2011). Pulse exposure of haematopoietic grafts to prostaglandin E2 in vitro facilitates engraftment and recovery. Cell Proliferation, 44, 22-29.

38. Broxmeyer, H. E., \& Pelus, L. M. (2014). Inhibition of DPP4/CD26 and $\mathrm{dmPGE}_{2}$ treatment enhances engraftment of mouse bone marrow hematopoietic stem cells. Blood Cells, Molecules \& Diseases, 53, 34-38.

39. Gul, H., Marquez-Curtis, L. A., Jahroudi, N., Lo, J., Turner, A. R., \& Janowska-Wieczorek, A. (2009). Valproic acid increases CXCR4 expression in hematopoietic stem/progenitor cells by chromatin remodeling. Stem Cells and Development, 18, 831-838.

40. Chaurasia, P., Gajzer, D. C., Schaniel, C., D’Souza, S., \& Hoffman, R. (2014). Epigenetic reprogramming induces the expansion of cord blood stem cells. The Journal of Clinical Investigation, 124, 2378-2395.

41. Janowska-Wieczorek, A., Majka, M., Kijowski, J., BajKrzyworzeka, M., Reca, R., Turner, A. R., et al. (2001). Plateletderived microparticles bind to hematopoietic stem/progenitor cells and enhance their engraftment. Blood, 98, 3143-3149.

42. Mantel, C. R., O'Leary, H. A., Chitteti, B. R., Huang, X., Cooper, S., Hangoc, G., et al. (2015). Enhancing hematopoietic stem cell transplantation efficacy by mitigating oxygen shock. Cell, 161, $1553-1565$.

43. Wysoczynski, M., Ratajczak, J., Pedziwiatr, D., Rokosh, G., Bolli, R., \& Ratajczak, M. Z. (2015). Identification of heme oxygenase 1
(HO-1) as a novel negative regulator of mobilization of hematopoietic stem/progenitor cells. Stem Cell Reviews, 11, 110-118.

44. Ko, K. H., Holmes, T., Palladinetti, P., Song, E., Nordon, R., O'Brien, T. A., et al. (2011). GSK-3 $\beta$ inhibition promotes engraftment of ex vivo-expanded hematopoietic stem cells and modulates gene expression. Stem Cells, 29, 108-118.

45. Dolnikov, A., Xu, N., Shen, S., Song, E., Holmes, T., Klamer, G., et al. (2014). GSK-3 $\beta$ inhibition promotes early engraftment of ex vivo-expanded haematopoietic stem cells. Cell Proliferation, 47, 113-123.

46. Freitas, A., Alves-Filho, J. C., Secco, D. D., Neto, A. F., Ferreira, S. H., Barja-Fidalgo, C., et al. (2006). Heme oxygenase/carbon monoxide-biliverdin pathway down regulates neutrophil rolling, adhesion and migration in acute inflammation. British Journal of Pharmacology, 149, 345-354.

47. Christopherson, K. W., Hangoc, G., Mantel, C. R., \& Broxmeyer, H. E. (2004). Modulation of hematopoietic stem cell homing and engraftment by CD26. Science, 305, 10001003.

48. Kimura, A., Ohmori, T., Kashiwakura, Y., Ohkawa, R., Madoiwa, S., Mimuro, J., et al. (2008). Antagonism of sphingosine 1phosphate receptor-2 enhances migration of neural progenitor cells toward an area of brain. Stroke, 39, 3411-3417.

49. Ren, H., Panchatcharam, M., Mueller, P., Escalante-Alcalde, D., Morris, A. J., \& Smyth, S. S. (2013). Lipid phosphate phosphatase (LPP3) and vascular development. Biochimica et Biophysica Acta, 1831, 126-132.

50. Broxmeyer, H. E., Kappes, F., Mor-Vaknin, N., Legendre, M., Kinzfogl, J., Cooper, S., et al. (2012). DEK regulates hematopoietic stem engraftment and progenitor cell proliferation. Stem Cells and Development, 21, 1449-1454.

51. Ratajczak, M. Z., Kucia, M., Jadczyk, T., Greco, N. J., Wojakowski, W., Tendera, M., et al. (2012). Pivotal role of paracrine effects in stem cell therapies in regenerative medicine: can we translate stem cell-secreted paracrine factors and microvesicles into better therapeutic strategies? Leukemia, 26, 1166-1173. 